\title{
Paracrine effects of TLR4-polarised mesenchymal stromal cells are mediated by extracellular vesicles
}

\author{
Marie-Theres Zeuner ${ }^{1}$, Ketan Patel $^{2,3}$, Bernd Denecke $^{4}$, Bernd Giebel ${ }^{5}$ and Darius Widera ${ }^{1 *}$ (D)
}

\begin{abstract}
Mesenchymal stromal cells (MSCs) are adult stem cells able to give rise to bone, cartilage and fat cells. In addition, they possess immunomodulatory and immunosuppressive properties that are mainly mediated through secretion of extracellular vesicles (EVs). In a previous issue of Journal of Translational Medicine, Ti and colleagues demonstrated that preconditioning of MSCs with bacterial lipopolysaccharides results in secretion of EVs that can polarise macrophages towards anti-inflammatory M2 phenotype. Moreover, the authors suggest that EVs of lipopolysaccharide (LPS)-treated MSCs are superior to EVs of untreated MSCs concerning their ability to support wound healing. Our commentary critically discusses parallel efforts of other laboratories to generate conditioned media from stem cells for therapeutic applications, and highlights impact and significance of the study of Ti et al. Finally, we summarise its limitations and spotlight areas that need to be addressed to better define the underlying molecular mechanisms.
\end{abstract}

Keywords: TLR4, LPS, Exosomes, Microvesicles, Extracellular vesicles, MSCs, Preconditioning

Multipotent MSCs can be easily raised from a variety of adult human tissues and organs, including bone marrow and adipose tissue [1], and possess immunomodulatory capabilities affecting the majority of immune cells [2]. As of Nov 2015, more than 540 clinical trials utilising MSCs have been registered in the database clinicaltrials.org [3].

It turns out that the therapeutic benefit of MSC-administration revealed in different proof of concept and clinical studies (for review see [4]) is frequently connected to paracrine/endocrine effects rather than to effects driven by the engraftment of MSCs into affected tissues and differentiation towards lost cell types [5-9].

Related to their proposed paracrine mode of action, several pre-clinical reports and a clinical treatment attempt of a Graft-versus-host disease (GvHD) patient provided evidence that MSCs exert their therapeutic functions via extracellular vesicles (EVs), such as

\footnotetext{
*Correspondence: d.widera@reading.ac.uk

1 Stem Cell Biology and Regenerative Medicine Group, Reading School of Pharmacy, University of Reading, Whiteknights Campus, PO Box 226, Reading RG6 6AP, UK

Full list of author information is available at the end of the article
}

exosomes and microvesicles [10-14]. Indeed, direct comparisons in mouse models of acute kidney failure and ischemic stroke revealed that MSCs and MSC-EVs exert comparable therapeutic effects $[11,12]$.

By definition, exosomes are small EVs $(\sim 70-150 \mathrm{~nm})$ of endosomal origin, while microvesicles (100-1000 nm) bud from the plasma membrane $[15,16]$. With the current technologies, nano-sized microvesicles and exosomes can hardly be discriminated at the experimental level. Consequently, the International Society of Extracellular Vesicles (ISEV) recommended to use, at least at the experimental level, the term EV rather than exosomes and microvesicles [17]. EVs are found in all body fluids and are secreted by almost all cell types, under physiological and pathological situations. Notably both, the content (a specific combination of different proteins, lipids, microRNAs as well as a small portion of mRNAs) and immunomodulatory features of EVs are cell type and context-dependent $[15,16,18-20]$.

Toll-like receptors (TLRs) are transmembrane receptors which play pivotal roles in the innate immune system by recognizing a wide spectrum of pathogen-associated 
molecules or pathogen-associated molecular patterns (PAMPs) as well as endogenous danger-/damage-associated molecular patterns (DAMPs) [21]. The toll-like receptor 4 (TLR4) recognises bacterial lipopolysaccharides (LPS) that depending on their chemotype can induce pro-inflammatory or immunosuppressive effects [22]. Importantly, also DAMPs are able to act as both, pro-inflammatory and immunosuppressive stimuli (reviewed in [23]). The binding of a ligand to TLR4 results in the activation of both, MyD88-dependent and independent signalling pathways. The MyD88-dependent signalling culminates in nuclear translocation of the proinflammatory transcription factor NF- $\mathrm{KB}$ dimers ( $\mathrm{p} 50$ and p65) and increased expression and secretion of proinflammatory cytokines (e.g., TNF- $\alpha$ ), whereas an active MyD88-independent pathway results in activation of IRF3 and synthesis of the anti-viral and anti-inflammatory interferon- $\beta$ [24].

MSCs express functional TLR4 $[25,26]$ but the consequences of its activation on proliferation, differentiation and migration seem to be largely dependent on the chemotype, purity and concentration of the ligand in addition to the duration of the exposure (reviewed in [27]). Besides such direct effects, MSCs can be efficiently polarised into a pro-inflammatory and anti-inflammatory phenotype by ligands of TLRs leading to respective changes in their immunomodulatory properties [28]. It has been recently reported that preconditioning of adipose-derived MSCs with low concentration of LPS $(0.5 \mathrm{ng} / \mathrm{ml}$, unspecified chemotype) improves the regenerative effects of MSCs-secretome in experimental hepatectomy in mice [29].

Here, we comment on the original research article by $\mathrm{Ti}$ and colleagues published recently in Journal of Translational Medicine [30]. In their study, the authors preconditioned umbilical cord-derived human MSCs with $100 \mathrm{ng} / \mathrm{mL}$ LPS (chemotype and purity not specified) for $48 \mathrm{~h}$ followed by collection of the supernatant and isolation of EVs via ultracentrifugation. They provide evidence that EVs of preconditioned MSCs are able to induce polarisation of the THP-1 monocyte cell line towards the anti-inflammatory M2 phenotype demonstrated by significant reductions in the levels of pro-inflammatory cytokine mRNA (IL-6, IL-1 and TNF- $\alpha$ ) and increased expression of anti-inflammatory transcripts IL-10 and TGF- $\beta$. Subsequently, Ti et al. were able to demonstrate that preconditioning of MSCs with LPS causes a change of EV miRNA cargo. They identified let-7b to be up-regulated most prominently among all of the 40 differentially expressed miRNAs. This is highly relevant since tumour suppressor miRNA let-7b is known to participate in regulation of cell proliferation and apoptosis and targets TLR4 [31, 32]. Similarly, in the study by Ti and colleagues, let-7b over-expression resulted in a significant decrease of TLR4 protein in THP-1 cells. Finally, in a rat model of diabetes, the application of EVs of preconditioned MSCs into cutaneous wound led to reduced infiltration of inflammatory cells and greatly improved the overall wound healing. Notably, EVs released by non-LPS treated MCS seem to transmit some beneficial effects in terms of tissue regeneration, albeit less pronounced than effects mediated by EVs released by LPS-treated cells. However, a major shortcoming of the study is the lack of quantitative data regarding the effects of EVs in the in vivo wound healing model hampering a direct comparison between the respective experimental groups. In general, diabetic wound healing models in rodents represent a potent tool in regenerative medicine and allow quantitative measurement of regeneration by assessing the epithelial gap and wound contraction [33]. Such quantitative parameters would greatly facilitate the evaluation of the effects of MSC-derived EVs on wound healing.

In summary, Ti and colleagues show that LPS modulates the secretome of umbilical MSCs. The authors demonstrate that EVs secreted by LPS-treated MSCs are anti-inflammatory and immunomodulatory. They further suggest that these effects are at least partly mediated by EV miRNA cargo. Finally, this study suggests that the EV fraction released from LPS-treated MSCs is enriched for the miRNA let-7b, which is known to target the TLR4/ NF-KB signalling pathway.

Although this study has high translational potential, there are several open questions that need to be addressed in future. Firstly, the study does not include LPS-treated MSCs as a control group in the wound healing model. Ideally, a direct comparison of the effects of transplanted EVs and the parental cells should be performed.

Secondly, the fate of LPS-treated MSCs remains an unresolved question. Since MSCs themselves can be polarised into pro- and anti-inflammatory phenotypes $[28,34]$, it is of major interest whether the pro- or antiinflammatory polarised MSCs secrete the anti-inflammatory EVs. In the present study, the stimulation of MSCs with LPS has been performed in serum-free medium. Notably, fetal calf serum (FCS) contains soluble CD14. As human MSCs lack the expression of CD14 $[35,36]$ that is believed to be necessary for induction of the MyD88 independent (anti-inflammatory) signalling pathway [37, 38 ], it could be presumed that most of the cells in this study underwent pro-inflammatory polarisation.

To investigate the ability of MSC-EVs to polarise macrophages, human monocytic cell line THP-1, originally isolated from peripheral blood of a 1-year-old male patient suffering from acute monocytic leukemia, has been applied [39]. Although widely used to study 
monocyte/macrophage functions, THP-1-derived macrophages differ from primary macrophages in terms of proliferation rate, expression pattern and notably also in terms of their sensitivity to LPS [40]. Thus, primary macrophages (e.g., derived from PBMCs) would represent a more appropriate cell population to test the EV-mediated polarisation.

Another major question to address is the role that let-7b plays in the process of diabetic wound closure. It would be of interest to determine whether the introduction of let-7b alone to the wound bed would sufficient to promote tissue regeneration by modulating the polarisation of macrophages. This would be an attractive therapeutic avenue since miRNA can be delivered to the lesion site using relatively safe vectors including the AAV family.

Another unresolved issue is the impact of different TLR4 ligands on the EV polarisation. In this context, it is noteworthy that the balance between the NF-kB signalling and the IRF3 activity is largely ligand-dependent [41]. Unfortunately, the study by $\mathrm{Ti}$ et al. does not provide information on the chemotype and purity of the LPS used, which both play a pivotal role in the downstream signalling (reviewed in [27]). In particular, standard LPS preparations activate TLR2 in addition to TLR4. In contrast to TLR4, TLR2 exclusively signals through the MyD88-dependent pathway and lacks the ability to activate IRF3. Thus, different LPS ligands induce fundamentally different downstream signalling cascades and could trigger different autocrine effects in MSCs.

Finally, since EVs contain a context-dependent and cell type specific combination of different proteins and lipids in addition to miRNAs, a holistic comparison of the cargo between EVs of LPS-treated and control MSCs could lead to better understanding of their regenerative properties.

\section{Conclusions}

Beyond its immediate relevance for the biology of MSCs, the study by $\mathrm{Ti}$ et al. establishes a perspective for improvement of cell-free therapy concepts in translational regenerative medicine. In future, EVs of in vitro "programmed" MSCs through inflammatory or damage signals could represent a promising and realistic alternative to stem cell transplantation. To exploit this potential, however, future research needs to unravel in detail their mode of action. Prior to therapeutic use it will be important to establish if pro- or anti-inflammatory polarised MSCs secrete the beneficial EVs. In addition, it will be interesting to treat MSCs with defined ultrapure LPS of different chemo- and serotypes prior to EV isolation. Such polarisation of MSCs via appropriate inflammatory signals could allow generating EVs with either pro- or anti-inflammatory properties. Notably, regeneration of various tissue including skeletal muscles require both, pro-inflammatory signals that drive proliferation and migration of progenitors as well as anti-inflammatory signals that regulate their differentiation and survival [42].

\section{Abbreviations}

AAV: adeno-associated virus; CD14: cluster of differentiation 14; DAMPs: damage associated molecular patterns; EVs: extracellular vesicles; FCS: foetal calf serum; IL: interleukin; IRF3: interferon regulatory factor 3; LPS: lipopolysaccharides; MSCs: mesenchymal stromal/stem cells; MyD88: myeloid differentiation primary response gene 88; NF-kB: nuclear factor 'kappa-light-chain-enhancer' of activated B-cells; PAMPs: pathogen-associated molecular patterns; TGF- $\beta$ : tumor growth factor beta; TLR2: toll-like receptor 2; TLR4: toll-like receptor 4; TNF: tumour necrosis factor.

\section{Authors' contributions}

All authors contributed to the writing of the manuscript. All authors read and approved the final manuscript.

\section{Author details}

${ }^{1}$ Stem Cell Biology and Regenerative Medicine Group, Reading School of Pharmacy, University of Reading, Whiteknights Campus, PO Box 226, Reading RG6 6AP, UK. ${ }^{2}$ School of Biological Sciences, University of Reading, Whiteknights Campus, Reading, UK. ${ }^{3}$ Freiburg Institute for Advanced Studies (FRIAS), University of Freiburg, Albertstr. 19, 79104 Freiburg, Germany. ${ }^{4}$ Interdisciplinary Center for Clinical Research Aachen (IZKF Aachen), RWTH Aachen University, Aachen, Germany. ${ }^{5}$ Institute for Transfusion Medicine, University Hospital Essen, University Duisburg-Essen, Essen, Germany.

\section{Acknowledgements}

$\mathrm{MZ}$ and DW are supported by a grant of the DFG (German Research Foundation, WI4318/2-1).

Commentary on Ti et al. J Transl Med (2015) 13:308; http://www.translationalmedicine.com/content/13/1/308

\section{Competing interests}

The authors declare that they have no competing interests.

Received: 18 November 2015 Accepted: 20 January 2016 Published online: 02 February 2016

\section{References}

1. Zuk PA, Zhu M, Ashjian P, De Ugarte DA, Huang Jl, Mizuno H, Alfonso ZC, Fraser JK, Benhaim P, Hedrick MH. Human adipose tissue is a source of multipotent stem cells. Mol Biol Cell. 2002;13:4279-95.

2. Wang Y, Chen X, Cao W, Shi Y. Plasticity of mesenchymal stem cells in immunomodulation: pathological and therapeutic implications. Nat Immunol. 2014;15:1009-16.

3. National Institutes of Health Clinical Trials database. https://www.clinicaltrials.gov

4. Sharma RR, Pollock K, Hubel A, McKenna D. Mesenchymal stem or stromal cells: a review of clinical applications and manufacturing practices. Transfusion. 2014;54:1418-37.

5. Lee RH, Pulin AA, Seo MJ, Kota DJ, Ylostalo J, Larson BL, Semprun-Prieto L, Delafontaine P, Prockop DJ. Intravenous hMSCs improve myocardial infarction in mice because cells embolized in lung are activated to secrete the anti-inflammatory protein TSG-6. Cell Stem Cell. 2009;5:54-63.

6. Zanotti L, Sarukhan A, Dander E, Castor M, Cibella J, Soldani C, Trovato AE, Ploia C, Luca G, Calvitti M, et al. Encapsulated mesenchymal stem cells for in vivo immunomodulation. Leukemia. 2013;27:500-3.

7. Timmers L, Lim SK, Arslan F, Armstrong JS, Hoefer IE, Doevendans PA, Piek J, El Oakley RM, Choo A, Lee CN, et al. Reduction of myocardial infarct size by human mesenchymal stem cell conditioned medium. Stem Cell Res. 2007;1:129-37.

8. Gnecchi M, He H, Liang OD, Melo LG, Morello F, Mu H, Noiseux N, Zhang $L$, Pratt RE, Ingwall JS, Dzau VJ. Paracrine action accounts for marked 
protection of ischemic heart by Akt-modified mesenchymal stem cells. Nat Med. 2005;11:367-8.

9. Gnecchi M, He H, Noiseux N, Liang OD, Zhang L, Morello F, Mu H, Melo LG, Pratt RE, Ingwall JS, Dzau VJ. Evidence supporting paracrine hypothesis for Akt-modified mesenchymal stem cell-mediated cardiac protection and functional improvement. FASEB J. 2006;20:661-9.

10. Lai RC, Arslan F, Lee MM, Sze NS, Choo A, Chen TS, Salto-Tellez M, Timmers $\mathrm{L}$, Lee CN, El Oakley RM, et al. Exosome secreted by MSC reduces myocardial ischemia/reperfusion injury. Stem Cell Res. 2010;4:214-22.

11. Bruno S, Grange C, Deregibus MC, Calogero RA, Saviozzi S, Collino F, Morando L, Busca A, Falda M, Bussolati B, et al. Mesenchymal stem cell-derived microvesicles protect against acute tubular injury. J Am Soc Nephrol. 2009;20:1053-67.

12. Doeppner TR, Herz J, Gorgens A, Schlechter J, Ludwig AK, Radtke S, de Miroschedji K, Horn PA, Giebel B, Hermann DM. Extracellular vesicles improve post-stroke neuroregeneration and prevent postischemic immunosuppression. Stem Cells Transl Med. 2015;4:1131-43.

13. Kordelas L, Rebmann V, Ludwig AK, Radtke S, Ruesing J, Doeppner TR, Epple M, Horn PA, Beelen DW, Giebel B. MSC-derived exosomes: a novel tool to treat therapy-refractory graft-versus-host disease. Leukemia. 2014;28:970-3.

14. Lener T, Gimona M, Aigner L, Borger V, Buzas E, Camussi G, Chaput N, Chatterjee D, Court FA, Del Portillo HA, et al. Applying extracellular vesicles based therapeutics in clinical trials_an ISEV position paper. J Extracell Vesicles. 2015;4:30087.

15. Ludwig AK, Giebel B. Exosomes: small vesicles participating in intercellular communication. Int J Biochem Cell Biol. 2012;44:11-5.

16. Yanez-Mo M, Siljander PR, Andreu Z, Zavec AB, Borras FE, Buzas El, Buzas K, Casal E, Cappello F, Carvalho J, et al. Biological properties of extracellular vesicles and their physiological functions. J Extracell Vesicles. 2015;4:27066

17. Raposo G, Stoorvogel W. Extracellular vesicles: exosomes, microvesicles, and friends. J Cell Biol. 2013;200:373-83.

18. The EVpedia database. http://evpedia.info

19. Exocarta -an exosome protein and RNA database. http://www.exocarta. org/

20. Montecalvo A, Larregina AT, Shufesky WJ, Stolz DB, Sullivan ML, Karlsson JM, Baty CJ, Gibson GA, Erdos G, Wang Z, et al. Mechanism of transfer of functional microRNAs between mouse dendritic cells via exosomes. Blood. 2012;119:756-66.

21. Ozinsky A, Underhill DM, Fontenot JD, Hajjar AM, Smith KD, Wilson CB, Schroeder L, Aderem A. The repertoire for pattern recognition of pathogens by the innate immune system is defined by cooperation between toll-like receptors. Proc Natl Acad Sci USA. 2000;97:13766-71.

22. Verghese MW, Snyderman R. Differential anti-inflammatory effects of LPS in susceptible and resistant mouse strains. J Immunol. 1981;127:288-93.

23. Piccinini AM, Midwood KS. DAMPening inflammation by modulating TLR signalling. Mediat Inflamm. 2010;2010:21.

24. Kawai T, Takeuchi O, Fujita T, Inoue J, Muhlradt PF, Sato S, Hoshino K, Akira S. Lipopolysaccharide stimulates the MyD88-independent pathway and results in activation of IFN-regulatory factor 3 and the expression of a subset of lipopolysaccharide-inducible genes. J Immunol. 2001;167:5887-94.

25. Liotta F, Angeli R, Cosmi L, Fili L, Manuelli C, Frosali F, Mazzinghi B, Maggi L, Pasini A, Lisi V, et al. Toll-like receptors 3 and 4 are expressed by human bone marrow-derived mesenchymal stem cells and can inhibit their T-cell modulatory activity by impairing Notch signaling. Stem Cells. 2008;26:279-89.

26. Hwa CH, Bae YC, Jung JS. Role of toll-like receptors on human adiposederived stromal cells. Stem Cells. 2006;24:2744-52.

27. Zeuner M, Bieback K, Widera D. Controversial role of toll-like receptor 4 in adult stem cells. Stem Cell Rev. 2015;11:621-34.

28. Waterman RS, Tomchuck SL, Henkle SL, Betancourt AM. A new mesenchymal stem cell (MSC) paradigm: polarization into a pro-inflammatory MSC1 or an immunosuppressive MSC2 phenotype. PLoS One. 2010;5:e10088.

29. Lee SC, Jeong HJ, Lee SK, Kim SJ. Lipopolysaccharide preconditioning of adipose-derived stem cells improves liver-regenerating activity of the secretome. Stem Cell Res Ther. 2015;6:75.

30. Ti D, Hao H, Tong C, Liu J, Dong L, Zheng J, Zhao Y, Liu H, Fu X, Han W. LPS-preconditioned mesenchymal stromal cells modify macrophage polarization for resolution of chronic inflammation via exosome-shuttled let-7b. J Transl Med. 2015;13:308.

31. Mayr C, Hemann MT, Bartel DP. Disrupting the pairing between let-7 and Hmga2 enhances oncogenic transformation. Science. 2007;315:1576-9.

32. Teng GG, Wang WH, Dai Y, Wang SJ, Chu YX, Li J. Let-7b is involved in the inflammation and immune responses associated with Helicobacter pylori infection by targeting toll-like receptor 4. PLoS One. 2013;8:e56709.

33. Sharma A, Singh AK, Warren J, Thangapazham RL, Maheshwari RK. Differential regulation of angiogenic genes in diabetic wound healing. J Invest Dermatol. 2006;126:2323-31.

34. Dumitru CA, Hemeda H, Jakob M, Lang S, Brandau S. Stimulation of mesenchymal stromal cells (MSCs) via TLR3 reveals a novel mechanism of autocrine priming. FASEB J. 2014;28:3856-66

35. Al-Nbaheen M, Vishnubalaji R, Ali D, Bouslimi A, Al-Jassir F, Megges M, Prigione A, Adjaye J, Kassem M, Aldahmash A. Human stromal (mesenchymal) stem cells from bone marrow, adipose tissue and skin exhibit differences in molecular phenotype and differentiation potential. Stem Cell Rev. 2013;9:32-43.

36. Secunda R, Vennila R, Mohanashankar AM, Rajasundari M, Jeswanth $S$, Surendran R. Isolation, expansion and characterisation of mesenchymal stem cells from human bone marrow, adipose tissue, umbilical cord blood and matrix: a comparative study. Cytotechnology. 2014;67:793-807.

37. Kagan JC, Su T, Horng T, Chow A, Akira S, Medzhitov R. TRAM couples endocytosis of toll-like receptor 4 to the induction of interferon-beta. Nat Immunol. 2008:9:361-8.

38. Zanoni I, Ostuni R, Marek LR, Barresi S, Barbalat R, Barton GM, Granucci F, Kagan JC. CD14 controls the LPS-induced endocytosis of toll-like receptor 4. Cell. 2011;147:868-80

39. Tsuchiya S, Yamabe M, Yamaguchi Y, Kobayashi Y, Konno T, Tada K. Establishment and characterization of a human acute monocytic leukemia cell line (THP-1). Int J Cancer. 1980;26:171-6.

40. Chanput W, Mes JJ, Wichers HJ. THP-1 cell line: an in vitro cell model for immune modulation approach. Int Immunopharmacol. 2014;23:37-45.

41. Netea MG, van Deuren M, Kullberg BJ, Cavaillon JM, Van der Meer JW. Does the shape of lipid A determine the interaction of LPS with toll-like receptors? Trends Immunol. 2002;23:135-9.

42. Tidball JG. Inflammatory processes in muscle injury and repair. Am J Physiol Regul Integr Comp Physiol. 2005;288:R345-53. 